\title{
Raman Detection of Bond Breaking and Making of a Chemisorbed Up-Standing Single Molecule at Single-Bond Level
}

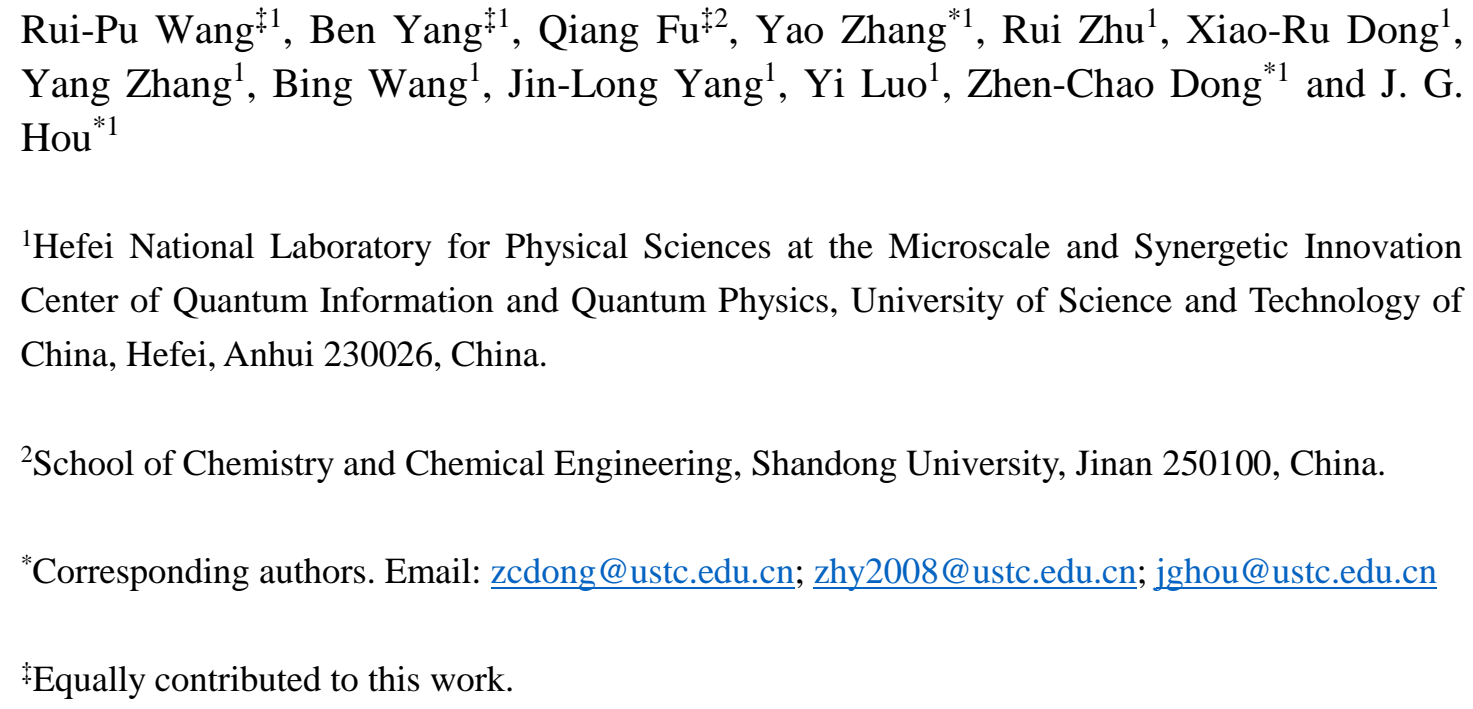
10 


\section{(1) Sample preparation.}

For the molecular sample with up-standing melamine configurations, melamine molecules (99\%, J\&K CHEMICAL LTD.) were thermally evaporated onto the $\mathrm{Cu}(100)$ surface held at room temperature through a Knudsen cell at $353 \mathrm{~K}$ for 2 min under ultrahigh vacuum (UHV) at $\sim 5.0 \times 10^{-8}$ torr. The sample was then transferred into the STM scanning stage, which was precooled to $6.2 \mathrm{~K}$ for measurements. The melamine sample with flat-lying configurations was obtained by in-situ deposition in the observation chamber with the $\mathrm{Cu}(100)$ substrate kept at $\sim 6 \mathrm{~K}$. The $\mathrm{Cu}(100)$ substrate was previously cleaned by cycles of argon ion sputtering and annealing.

\section{(2) STM and TERS measurements.}

All STM imaging and TERS experiments were performed on a custom optical-STM system operating under a UHV chamber (base pressure $<1.5 \times 10^{-10}$ torr) and at liquid-helium cryogenic conditions $(\sim 6.2 \mathrm{~K}) .{ }^{1}$ The thermal drift is about $50 \mathrm{pm} / \mathrm{h}$ at $\sim 6.2 \mathrm{~K}$ under laser illumination, which is almost negligible for the measurement time used in the present work. Electrochemically etched sliver tips were used in all our experiments. The tip apex was cleaned by electron-bombarding and argon-ion sputtering inside UHV, followed by further atomistic modification through tip indentation on a clean $\mathrm{Ag}(100)$ surface to achieve TERS-active status for efficient light coupling. Then the TERS-active Ag tip was used to conduct TERS spectral and mapping measurements on the melamine/ $\mathrm{Cu}(100)$ sample. Once a Ag tip lost its TERS activity, atomistic modifications were carried out again on a clean $\operatorname{Ag}(100)$ surface to recover its TERS-active status. A single-longitude-mode diode-pumped laser at $532 \mathrm{~nm}$ (Cobolt 0532-08-11-0160-100) was used to provide a linearly polarized laser beam (>100:1) for Raman excitation. A round continuously variable metallic neutral density filter was used to adjust the laser output power. Two reflective mirrors were used to provide freedoms for optical alignment. A half-wave plate was used to achieve desired p-polarization for incident laser. The laser beam was introduced into the UHV chamber via a quartz viewport after reflected by a 30:70 (R:T) beam splitter. Another beam splitter, which was removed during Raman measurements, was positioned in the optical collection path to help monitor the focusing of the laser beam into the tunnel junction with a video camera. The collimated beam was re-focused by an aspheric lens $(f=12.4 \mathrm{~mm}, \mathrm{NA}=0.46)$ into the tip-substrate junction with an angle of $60^{\circ}$ from the surface normal. The diameter of the laser spot on the sample surface was about $30 \mu \mathrm{m}$. The Raman scattered light was collected by the same lens, transmitted through the 30:70 (R:T) beam splitter, filtered by an edge filter to remove residual laser light, and finally fiber-coupled to a spectrometer using a slit size of $100 \mu \mathrm{m}$. The Raman signal light was dispersed by a 600 grooves $/ \mathrm{mm}$ grating and detected by a liquid-nitrogen cooled charge-coupled-device (CCD) (Princeton Instrument). The laser power used in our TERS experiments was about $0.2 \mathrm{~mW}$. The spectral resolution was about $17 \mathrm{~cm}^{-1}$, limited by the instrumental resolution as a result of a balance with the photon collection efficiency and the detection of Raman photons with a reasonable signal-to-noise ratio. The STM operation was controlled by Nanonis-SPECS electronics. All STM imaging and single-point TERS spectral measurements presented here were carried out in a constant-current mode with the sample biased. The TERS mapping was carried out in a constant-height mode through a synchronization function between the STM controller and CCD camera, acquiring a spectrum at each pixel during scanning. The constant-height mode was adopted for TERS mapping to avoid the disturbance to the molecular structure caused by the tip-height modulation during scanning. All Raman spectra 
presented here were not corrected for the wavelength-dependent sensitivity of photon detection systems.

\section{(3) Theoretical calculations.}

Our density functional theory (DFT) calculations are performed by utilizing the Vienna ab initio simulation package (VASP). ${ }^{2-3}$ The projector augmented wave (PAW) approach ${ }^{4}$ is employed and the energy cutoff of the plane-wave basis set is set to $500 \mathrm{eV}$. The exchange-correlation interactions between electrons are described by the optPBE-vdW functional, ${ }^{5-6}$ which explicitly includes the effect of van der Waals forces between atoms. The $\mathrm{Cu}(100)$ surface is modeled by periodically repeated slabs consisting of five $\mathrm{Cu}$ layers separated by a $20-\AA$ thick vacuum region. Here, the experimental $\mathrm{Cu}$ lattice constant of $3.61 \AA$ and a $6 \times 6$ surface periodicity are used. The Brillouin zone is sampled by using a $\Gamma$-centered $2 \times 3 \times 1 k$-grid. Dipole corrections on both the electrostatic potential and the total energy are added along the perpendicular direction. The atomic coordinates of the top three $\mathrm{Cu}$ layers as well as the melamine molecule are relaxed until the maximum force is less than $0.02 \mathrm{eV} / \mathrm{A}$. For the calculation of an isolated melamine molecule, the molecule is placed within a $22 \AA \times 20 \AA \times 16 \AA$ supercell, and only the $\Gamma$ point is used for the Brillouin zone sampling. The Raman spectra of the adsorbed molecule on the $\mathrm{Cu}(100)$ surface are simulated by using the quasi-analytical approach, ${ }^{7}$ while the Raman spectrum of an isolated melamine molecule in free space is obtained from the Python script for the calculations of the polarizability derivatives. ${ }^{8}$

\section{S2. Analysis of full Raman spectromicroscopic images}

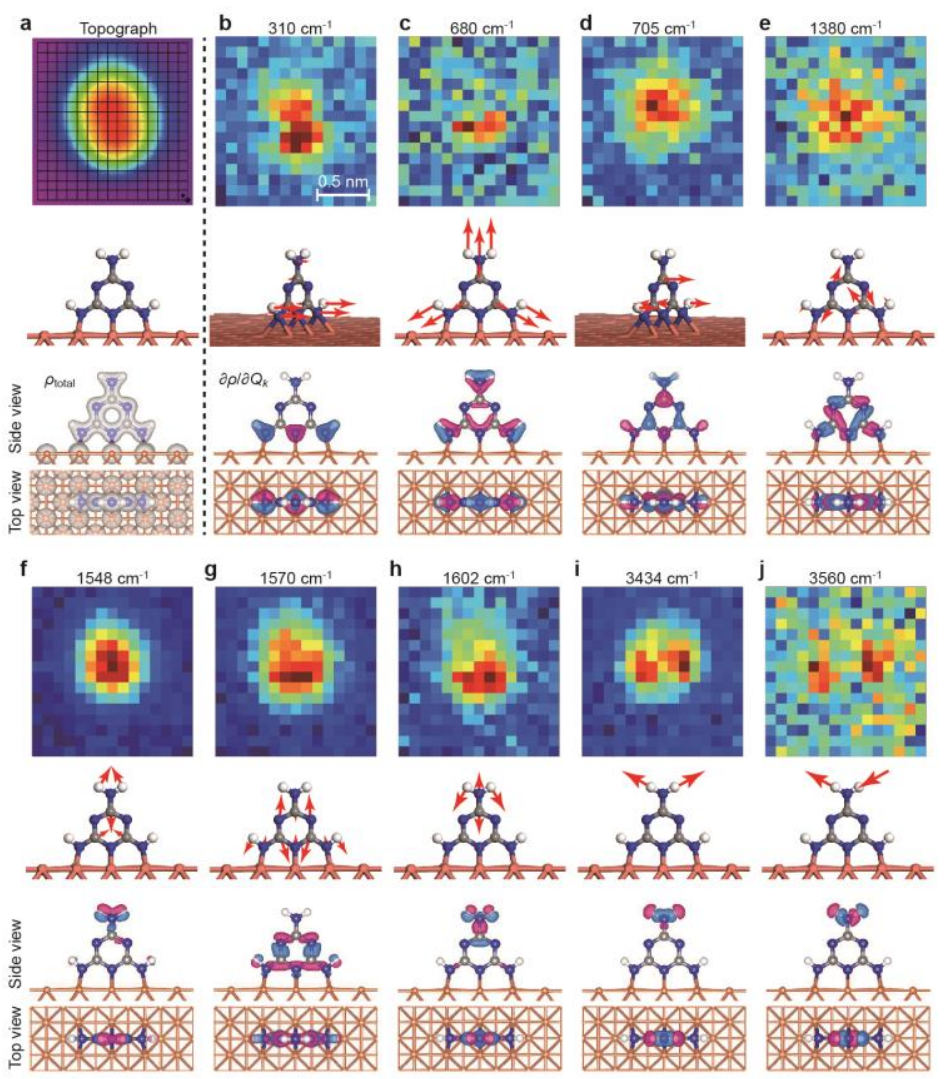

Fig. S1. TERS mapping images for an up-standing dehydrogenated melamine on $\mathbf{C u}(\mathbf{1 0 0})$. a, Top: STM image $(-0.05 \mathrm{~V}, 2 \mathrm{pA})$ with a $16 \times 15$ grid overlapped to show the mapping region. Middle: Adsorption configuration. Bottom: Side and top views of total electron density $\left(\rho_{\text {total }}\right) . \mathbf{b}-\mathbf{j}$, Top: 
Representative TERS spatial mapping images acquired at constant height mode with the height regulated at the set-point tunneling condition of $-0.05 \mathrm{~V}$ and $5 \mathrm{nA}$ above the molecular center $(1.6 \mathrm{~nm}$ $\times 1.5 \mathrm{~nm}, 16 \times 15$ pixels, $15 \mathrm{~s}$ per pixel). Middle: Schematic of molecular vibrations for the Raman modes, with the atomic displacements highlighted by red arrows. Bottom: Side and top views of the calculated spatial distribution of electronic density derivatives $\partial \rho / \partial Q_{k}$ for the vibrational mode. The blue and red colors denote different phase relations of the density derivatives for different vibrational modes.

The up-standing configuration can be further justified by full Raman spectromicroscopic images since a panoramic view of the spatial distributions of various vibrational modes can provide more comprehensive information on the adsorption configuration. ${ }^{1,9}$ Figure S1 shows the TERS mapping images for the nine Raman peaks labelled in main-text Fig. 1c. The Raman intensity of each vibrational mode is correlated with the polarizability derivative, namely, $I_{\text {Raman }} \propto \partial \alpha / \partial Q_{k}=-\int\left[\partial\left(\partial \rho / \partial Q_{k}\right) / \partial \mathbf{E}\right] \mathbf{r} d \mathbf{r}$, where $\rho$ is the electronic density, $Q_{k}$ denotes the normal mode coordinate, $\mathbf{r}$ is the Cartesian coordinate, and $\mathbf{E}$ is the local field. Therefore, the real-space perturbation of molecular vibrations (i.e., mode amplitudes and phases) on the electronic densities can provide insights into the spatial distribution of Raman mapping images.

The density functional theory (DFT) calculations of the derivative of electronic densities $\left(\partial \rho / \partial Q_{k}\right)$ are performed by Vienna ab initio simulation package (VASP). ${ }^{2-3}$ The molecular structure adsorbed on $\mathrm{Cu}(100)$ is first optimized and the molecular vibrations are calculated based on the optimized structure. Then the geometry of the molecule upon vibration is distorted according to the atomic displacement (with a scaling factor 0.1) obtained for each vibrational mode, and the spatial distribution of the total electronic density ptotal is calculated for each distorted geometry. The derivative of electronic density $\partial \rho / \partial Q_{k}$ for the $k$-th mode is obtained numerically via $\partial \rho / \partial Q_{k}=\left(\rho_{Q_{k}}-\rho_{Q_{\bar{k}}}\right) / 2 \Delta Q_{k}$, where the sign “+/-" denotes that the molecular geometry is distorted with the positive/negative atomic displacements.

The spatial distributions of $\partial \rho / \partial Q_{k}$ for different vibrational modes are plotted in Fig. S1 below each mapping image. Overall speaking, the more localized a molecular vibration, the more confined the spatial distribution of its electronic density derivative. Furthermore, such perturbed electronic density would be polarized by the local plasmonic field, resulting in the vibration-perturbed scattering (i.e. the Raman scattering) of a single molecule. For example, the Raman peaks at $3434 \mathrm{~cm}^{-1}$ and $3560 \mathrm{~cm}^{-1}$ are related to the symmetric and anti-symmetric stretching vibrations of top two $\mathrm{N}-\mathrm{H}$ bonds (See movie 1 for vibration animation). Thus, the spatial distribution of $\partial \rho / \partial Q_{k}$ is mainly localized on the top amino group, but different phase relations between the two $\mathrm{N}-\mathrm{H}$ bonds, i.e., in-phase for Fig. S1i and out-of-phase for Fig. S1j, will result in the constructive and destructive interference of the Raman signals, generating different patterns in corresponding Raman images, as observed experimentally. For the mode at $~ 3560$ $\mathrm{cm}^{-1}$, the mapping image exhibits a feature of "two bright dots" roughly along the $\mathrm{H}-\mathrm{N}-\mathrm{H}$ direction with little Raman intensities at the center, indicating an out-of-phase destructive interference associated with two anti-symmetric $\mathrm{N}-\mathrm{H}$ stretching vibrations. While for the $\sim 3434$ $\mathrm{cm}^{-1}$ mode, the corresponding Raman peak has considerable intensities around the center position, exhibiting an in-phase constructive interference for the symmetric stretching vibration of two $\mathrm{N}-\mathrm{H}$ bonds. Such spatial feature is consistent with the line-trace TERS data shown previously in 
main-text Fig. 2. Note that the mode at $\sim 3326 \mathrm{~cm}^{-1}$ associated with the stretching vibration of the bottom $\mathrm{N}-\mathrm{H}$ bonds was not observed under the mapping condition because of the tip being far away and the resultant low signal-to-noise ratio in Raman intensities.

Similar analyses on the density derivatives can be also applied to other vibrational modes. For example, the vibrational modes at 1548,1570 and $1602 \mathrm{~cm}^{-1}$ for the melamine molecule are associated with the $\mathrm{C}-\mathrm{N}$ stretching motions or $-\mathrm{NH}_{2}$ deformations ${ }^{10}$ (see Movie 1 for vibrational animation). Therefore, the whole skeleton of the melamine molecule would be always influenced, resulting in a dispersive "bright spot" feature for all three TERS mapping images. The normal Raman peak at $674 \mathrm{~cm}^{-1}$ is associated with the overall vibration of the amino groups relative to the cyanuric ring, which becomes weaker in the TERS setup (and is also slightly blue-shifted to $680 \mathrm{~cm}^{-1}$, as shown in Fig. S1c) presumably due to the bonding constraints from the substrate upon dehydrogenation. Surprisingly, this mode is highly localized in space, demonstrating a spatial resolution down to $\sim 0.2 \mathrm{~nm}$, which is likely due to the dominant contribution from the highly localized vertical vibration of the top $-\mathrm{NH}_{2}$ group as a whole. Interestingly, for the Raman peak at $310 \mathrm{~cm}^{-1}$, the TERS mapping image exhibits a different spatial feature showing an roughly "dumbbell" shape perpendicular to the molecular plane, which may be related to the out-of-plane vibration of the $\mathrm{N}$ atoms bonded to the $\mathrm{Cu}$ substrate. All these features in vibrational-mode imaging confirm the up-standing configuration of the adsorbed melamine molecule involving a dehydrogenation process.

\section{S3. Probing tip-induced deformation of top N-H bonds via TERS spectral evolution}
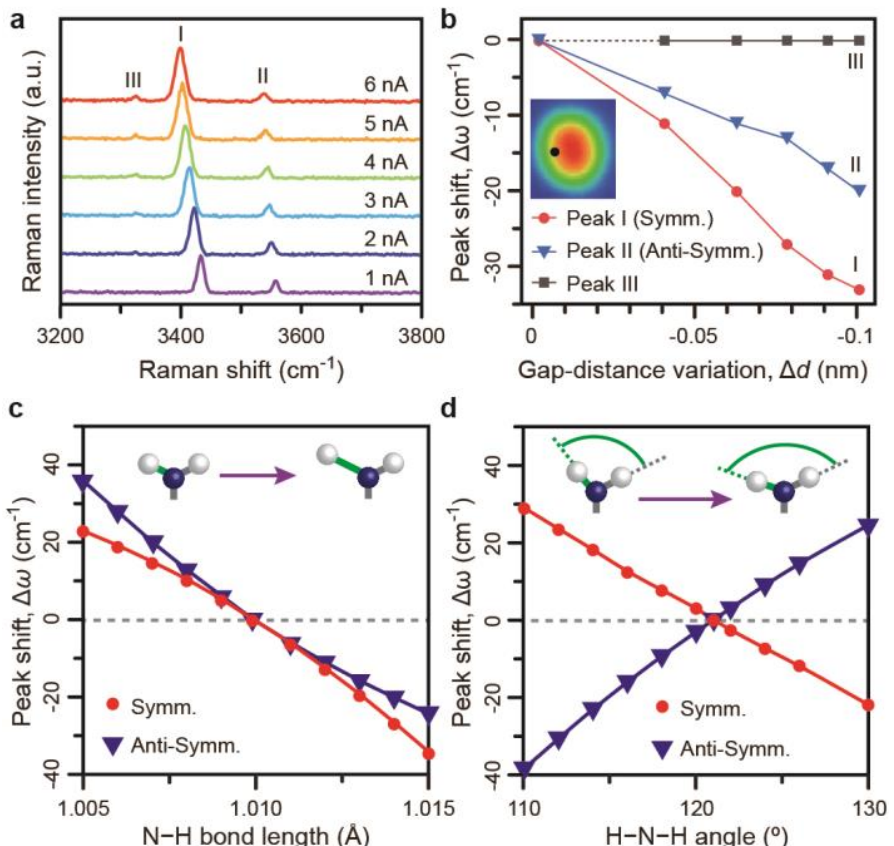

\section{Fig. S2. Probing tip-induced deformation of top $\mathrm{N}-\mathrm{H}$ bonds via TERS spectral evolution. a,} Dependence of Raman peak shifts on tunneling currents over $3000-3800 \mathrm{~cm}^{-1}$, with the TERS spectra $(-0.02 \mathrm{~V}, 60 \mathrm{~s})$ acquired at the position marked in the inset image in (b). The tunneling current is changed from $1 \mathrm{nA}$ to $6 \mathrm{nA}$. b, Relative peak shift $\Delta \omega$ for the three identified Raman peaks as a function of gap-distance variations $(\Delta d)$. c,d, Theoretical simulation of peak shifts of $\mathrm{N}-\mathrm{H}$ symmetric and anti-symmetric stretching modes against $\mathrm{N}-\mathrm{H}$ bond lengths and $\mathrm{H}-\mathrm{N}-\mathrm{H}$ bond angles. The $\mathrm{N}-\mathrm{H}$ 
bond at one side is stationary during the changes. The red and blue solid lines are drawn as a guide to the eye. The horizontal gray dashed line marks the zero-shift position. The schematics of changes in bond lengths and angles are sketched as insets.
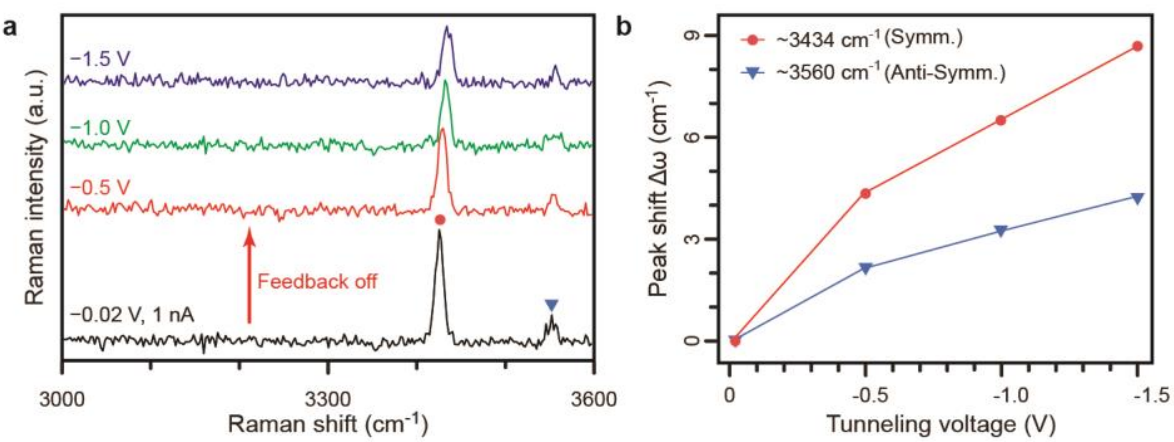

Fig. S3. Bias-dependent Raman peak shifts at a fixed gap distance. a, TERS spectra ( $1 \mathrm{nA}, 60 \mathrm{~s})$ as a function of bias voltages at a fixed gap distance regulated at the set-point tunneling condition of $-0.02 \mathrm{~V}$ and $1 \mathrm{nA}$ (namely in the constant-height mode). b, Measured Raman peak shifts at different tunneling voltages for the symmetric and anti-symmetric $\mathrm{N}-\mathrm{H}$ bond stretching vibrational modes at $\sim 3434 \mathrm{~cm}^{-1}$ and $\sim 3560 \mathrm{~cm}^{-1}$, respectively.

As shown in main-text Fig. 2a, the disturbance caused by spatially varying tip-molecule interactions on the top amino group could result in evident shifts of certain Raman peaks, especially for the symmetric and anti-symmetric $\mathrm{N}-\mathrm{H}$ stretching vibrations. By tracking the spectral evolution of the TERS spectra at different vertical tip-molecule distances, we can quantitatively investigate the deformation of top $\mathrm{N}-\mathrm{H}$ bonds caused by such tip-molecule interactions, by combining with theoretical simulations. As shown in Fig. S2a, the Raman peaks in the region of $3000-3800 \mathrm{~cm}^{-1}$ for $\mathrm{N}-\mathrm{H}$ stretching vibrations are found to shift to red continuously as the tip approaches to the molecule from the top, particularly evident for peaks I and II associated with the top $-\mathrm{NH}_{2}$ group. Peak III at $\sim 3326 \mathrm{~cm}^{-1}$ is very weak and only starts to emerge when the tip is relatively close to the molecule. This, together with the indiscernible peak shift, again suggests that peak III can be attributed to the stretching vibrations of the bottom $\mathrm{N}-\mathrm{H}$ bonds of the adsorbed molecule since they are hardly affected by the tip far away (about $3.7 \AA$ at $6 \mathrm{nA}$, see Fig. S4 for details). Fig. S2b shows the relative peak shifts $(\Delta \omega)$ of these three peaks at $\sim 3326$ $\mathrm{cm}^{-1}, \sim 3434 \mathrm{~cm}^{-1}$ and $\sim 3560 \mathrm{~cm}^{-1}$ as a function of the changes in the gap distance $(\Delta d)$, which can be obtained by recording the tip heights at different tunneling currents (Fig. S4). The largest red-shift is observed for the Raman mode at $\sim 3434 \mathrm{~cm}^{-1}$ associated with the symmetric stretching vibration of the two $\mathrm{N}-\mathrm{H}$ bonds at the top, which is as large as $\sim 33 \mathrm{~cm}^{-1}$ for a small change in the gap distance $(\Delta d \sim 1.0 \AA$ ) when tunneling currents are changed from $1 \mathrm{nA}$ to $6 \mathrm{nA}$. Interestingly, for the anti-symmetric stretching mode of the same $\mathrm{N}-\mathrm{H}$ bonds at $\sim 3560 \mathrm{~cm}^{-1}$, the amount of red-shifts is only $\sim 20 \mathrm{~cm}^{-1}$, still quite significant but much smaller than the symmetric stretching mode.

There are two possibilities to cause the peak shifts upon tip approaching: one is the deformation of related chemical bonds due to ever-increasing tip-molecule interactions, the other is the Stark effect induced by the ever-increasing local electric field in the nanocavity. The latter possibility can be excluded by examining the evolution of TERS spectra taken at a constant tip height but with varying bias voltages. As shown in Fig. S3, for a tip height regulated at the tunneling 
condition of $-0.02 \mathrm{~V}$ and $1 \mathrm{nA}$, field-induced peak shifts over a bias range from $-0.02 \mathrm{~V}$ to $-1.5 \mathrm{~V}$ are very small $\left(\sim 3 \mathrm{~cm}^{-1}\right.$ for the anti-symmetric mode and $\sim 9 \mathrm{~cm}^{-1}$ for the symmetric mode), even though the field strength is increased by about 75 times, much larger than the field increase presented in Fig. S2a when the tunneling current is changed from $1 \mathrm{nA}$ to $6 \mathrm{nA}$. More importantly, the peak is shifted to blue with increasing field strengths, just opposite to the red-shifting trend observed in Fig. S2a. Therefore, the Stark effect is definitely not the mechanism causing the red shifts observed in Fig. S2a.

In order to investigate the influence of tip-molecule interactions on the deformation of related chemical bonds and resultant peak shifts, we simulate the frequency shifts of symmetric and anti-symmetric vibrational modes of two $\mathrm{N}-\mathrm{H}$ bonds within the top amino group of the adsorbed dehydrogenated melamine molecule. Such simulation is realized by manually tuning the $\mathrm{N}-\mathrm{H}$ bond length $\left(d_{\mathrm{N}-\mathrm{H}}\right)$ and $\mathrm{H}-\mathrm{N}-\mathrm{H}$ bond angle $\left(\theta_{\mathrm{H}-\mathrm{N}-\mathrm{H}}\right)$ in the top $-\mathrm{NH}_{2}$ group with respect to the structure optimized on the substrate. The calculated results are shown in Fig. S2c and Fig. S2d, respectively. The simultaneous changes in both the bond length and angle result in different amplitudes of Raman peak shifts for the symmetric and anti-symmetric vibrations: the former adds up while the latter cancels out to some extent, which explains why the observed red shift for the symmetric mode $\left(\sim 33 \mathrm{~cm}^{-1}\right)$ is much larger than the anti-symmetric mode $\left(\sim 20 \mathrm{~cm}^{-1}\right)$. As a result, the energy spacing between these two modes is enlarged when the $\mathrm{H}-\mathrm{N}-\mathrm{H}$ bond angle is increased (Fig. S2a and Fig. S2b), which is an indication of strengthened vibrational coupling between the two $\mathrm{N}-\mathrm{H}$ bonds.

It can be found that the frequency shifts almost follow the linear dependence with varying bond lengths and angles, especially near the optimized molecular structural parameter $d_{\mathrm{N}-\mathrm{H}}^{0}=1.010 \AA$ and $\theta_{\mathrm{H}-\mathrm{N}-\mathrm{H}}^{0}=121.02^{\circ}$. Therefore, the symmetric and anti-symmetric vibration frequencies can be expressed in a general way as

$\Delta \omega_{\mathrm{symm}}\left(d_{\mathrm{N}-\mathrm{H}}, \theta_{\mathrm{H}-\mathrm{N}-\mathrm{H}}\right)=A\left(d_{\mathrm{N}-\mathrm{H}}-d_{\mathrm{N}-\mathrm{H}}^{0}\right)+B\left(\theta_{\mathrm{H}-\mathrm{N}-\mathrm{H}}-\theta_{\mathrm{H}-\mathrm{N}-\mathrm{H}}^{0}\right)$,

$\Delta \omega_{\text {anti-symm }}\left(d_{\mathrm{N}-\mathrm{H}}, \theta_{\mathrm{H}-\mathrm{N}-\mathrm{H}}\right)=C\left(d_{\mathrm{N}-\mathrm{H}}-d_{\mathrm{N}-\mathrm{H}}^{0}\right)+D\left(\theta_{\mathrm{H}-\mathrm{N}-\mathrm{H}}-\theta_{\mathrm{H}-\mathrm{N}-\mathrm{H}}^{0}\right)$,

where $\Delta \omega_{\text {symm }}$ and $\Delta \omega_{\text {anti-symm }}$ are the calculated vibrational frequency shifts for the symmetric and anti-symmetric stretching vibrations of two $\mathrm{N}-\mathrm{H}$ bonds in the atop amino group with respect to the optimized structure of adsorbed melamine molecule. By fitting the calculated frequency shift with the linear curves in Fig. S2c and Fig. S2d, we can obtain the fitting parameters as $A=-67.15$ $\mathrm{cm}^{-1} / \mathrm{pm}, B=-2.53 \mathrm{~cm}^{-1} /$ degree, $C=-47.63 \mathrm{~cm}^{-1} / \mathrm{pm}$ and $D=2.48 \mathrm{~cm}^{-1} /$ degree. By substituting the experimentally obtained Raman frequencies of symmetric and anti-symmetric modes (Fig. $\mathrm{S} 2 \mathrm{~b}$ ) into above equations, the fitted values of the changes in $\mathrm{N}-\mathrm{H}$ bond lengths and $\mathrm{H}-\mathrm{N}-\mathrm{H}$ bond angles are listed in Table S1. In other words, based on the observed peak shifts, we can estimate quantitatively the possible bond lengths and angles at each gap distance when the tip approaches the molecule (Fig. S2a). Specifically, upon such a tip approaching, an overall increase of $\sim 0.005 \AA$ in the $\mathrm{N}-\mathrm{H}$ bond length and $\sim 2.44^{\circ}$ in the $\mathrm{H}-\mathrm{N}-\mathrm{H}$ angle is expected to occur by referring to the optimized molecular structure with $d_{\mathrm{N}-\mathrm{H}}=1.010 \AA$ and $\angle_{\mathrm{H}-\mathrm{N}-\mathrm{H}}=121.02^{\circ}$.

Similar mechanism can be also applied to explain the peak shifts observed in main-text Fig. 2a, whereas the tip approaches laterally to the molecule, the peak positions are red-shifted due to growing interactions between the tip and the top $\mathrm{N}-\mathrm{H}$ bonds. The ability of TERS to detect the 
dynamic variations in the local bonding geometry (i.e., bond length and angle) is certainly a unique advantage of the technique itself and may be used to explore bond-bond or orbital-orbital interactions.

\section{Table S1.}

Fitted values of $\mathrm{N}-\mathrm{H}$ bond lengths $\left(d_{\mathrm{N}-\mathrm{H}}\right)$ and $\mathrm{H}-\mathrm{N}-\mathrm{H}$ bond angles $\left(\theta_{\mathrm{H}-\mathrm{N}-\mathrm{H}}\right)$ from experimental results.

\begin{tabular}{|c|c|c|c|c|}
\hline \multirow{2}{*}{$\begin{array}{c}\text { Tunneling } \\
\text { current }(\mathrm{nA})\end{array}$} & \multicolumn{2}{|c|}{ Peak shift $\left(\mathrm{cm}^{-1}\right)$} & $\begin{array}{c}\mathrm{N}-\mathrm{H} \text { bond } \\
\text { length, } d_{\mathrm{N}-\mathrm{H}}(\AA)\end{array}$ & $\begin{array}{c}\mathrm{H}-\mathrm{N}-\mathrm{H} \text { bond } \\
\text { angle, } \theta_{\mathrm{H}-\mathrm{N}-\mathrm{H}}\left({ }^{\circ}\right)\end{array}$ \\
\cline { 2 - 3 } & Symm. mode & Anti-symm. mode & 1.0099 & 121.021 \\
\hline 1 & 0 & 0 & 1.0115 & 121.208 \\
\hline 2 & -11 & -7 & 1.0126 & 121.765 \\
\hline 3 & -20 & -11 & 1.0134 & 122.459 \\
\hline 4 & -27 & -13 & 1.0141 & 122.187 \\
\hline 5 & -31 & -17 & 1.0145 & 121.817 \\
\hline 6 & -33 & -20 & \\
\hline
\end{tabular}

S4. Tip height measurements and estimation on the detection depth $\left(d_{\mathrm{depth}}\right)$ of TERS

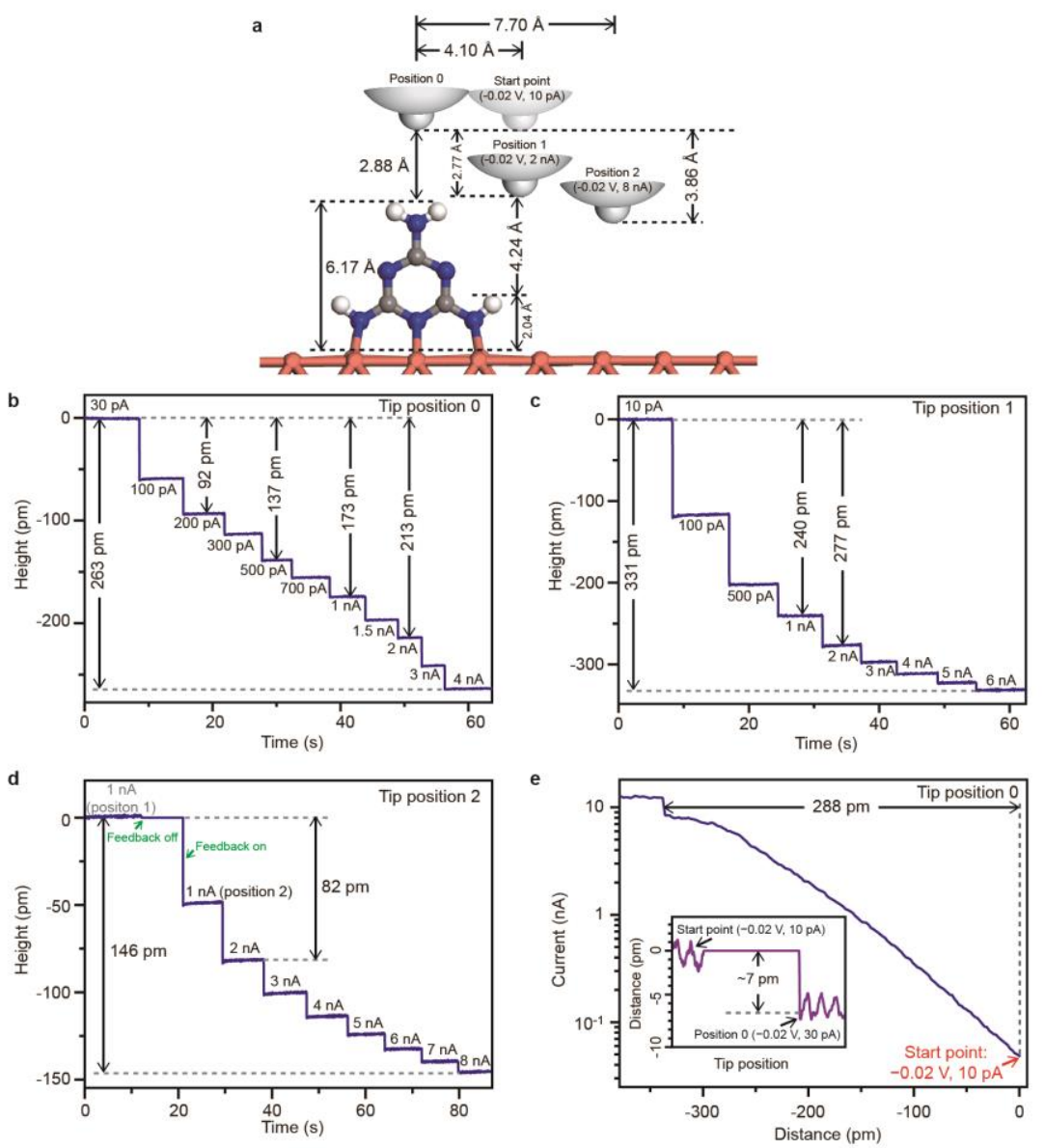

Fig. S4. Tip height measurements. a, Schematic of different tip heights in the STM junction. b, The variation of tip heights at position 0 for the tunneling condition at $-0.02 \mathrm{~V}$ with different currents from $30 \mathrm{pA}$ to $4 \mathrm{nA}$. c, The variation of tip heights at position 1 for the tunneling condition at $-0.02 \mathrm{~V}$ with different currents from $10 \mathrm{pA}$ to $6 \mathrm{nA}$. d, The variation of tip heights at position 2 for the tunneling 
condition at $-0.02 \mathrm{~V}$ with different currents from $1 \mathrm{nA}$ to $8 \mathrm{nA}$. The tip was initially located at position 1 at the condition $(-0.02 \mathrm{~V}, 1 \mathrm{nA})$ and then moved laterally to position 2 by turning off the feedback. Then the feedback was turned on to record the tip height changes with increasing tunneling currents. e, The tip trajectory during the approaching to the top center of the adsorbed melamine molecule at tip position 0 to the contact point from a reference start point. The tip was initially located at position 1 at the condition $(-0.02 \mathrm{~V}, 10 \mathrm{pA})$ and then moved laterally to position 0 above the molecular center by turning off the feedback. The inset shows the distance variation by $0.07 \AA$ when the tip moves from the start point at position $1(-0.02 \mathrm{~V}, 10 \mathrm{pA})$ to the tunneling condition at position $0(-0.02 \mathrm{~V}, 30 \mathrm{pA})$, giving rise to a tip-molecule distance of $\sim 2.81 \AA(2.88 \AA-0.07 \AA)$ at this condition. The distances at other tunneling currents can be obtained from the labelled distances in (b) accordingly (main-text Fig.3b). When the tip is located at position 1 with the tunneling condition of $-0.02 \mathrm{~V}$ and $2 \mathrm{nA}$, the tip is $\sim 0.11 \AA(2.88 \AA-2.77 \AA)$ vertically above the top of molecule. The detection depth $\left(\boldsymbol{d}_{\text {depth }}\right)$, defined by the onset distance to detect the Raman signal of the bottom N-H bond, can be estimated as $4.24 \AA(6.17 \AA+2.88 \AA-2.77 \AA-2.04 \AA)$. When the tunneling current is increased to $6 \mathrm{nA}$, the tip is $\sim 0.43 \AA(3.31 \AA-2.88 \AA)$ vertically below the top of molecule and $\sim 3.70 \AA(6.17 \AA+2.88 \AA-3.31 \AA$ $-2.04 \AA$ ) above the $\mathrm{N}-\mathrm{H}$ bond at the bottom. When the tip is located at position 2 with the tunneling condition of $-0.02 \mathrm{~V}$ and $2 \mathrm{nA}$, the tip is $\sim 0.34 \AA(0.82 \AA+2.40 \AA-2.88 \AA=3.22 \AA-2.88 \AA)$ vertically below the top of molecule. When the tunneling current is increased to $8 \mathrm{nA}$, the tip is $\sim 0.98$ $\AA(1.46 \AA+2.40 \AA-2.88 \AA=3.86 \AA-2.88 \AA)$ vertically below the top of molecule.

\section{S5. Bond-related analyses for optimized chemical structures and simulated Raman spectra before and after tautomerization}

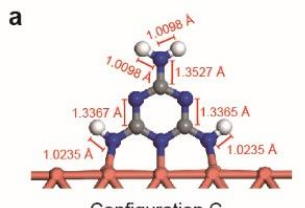

Configuration $\mathrm{C}_{0}$
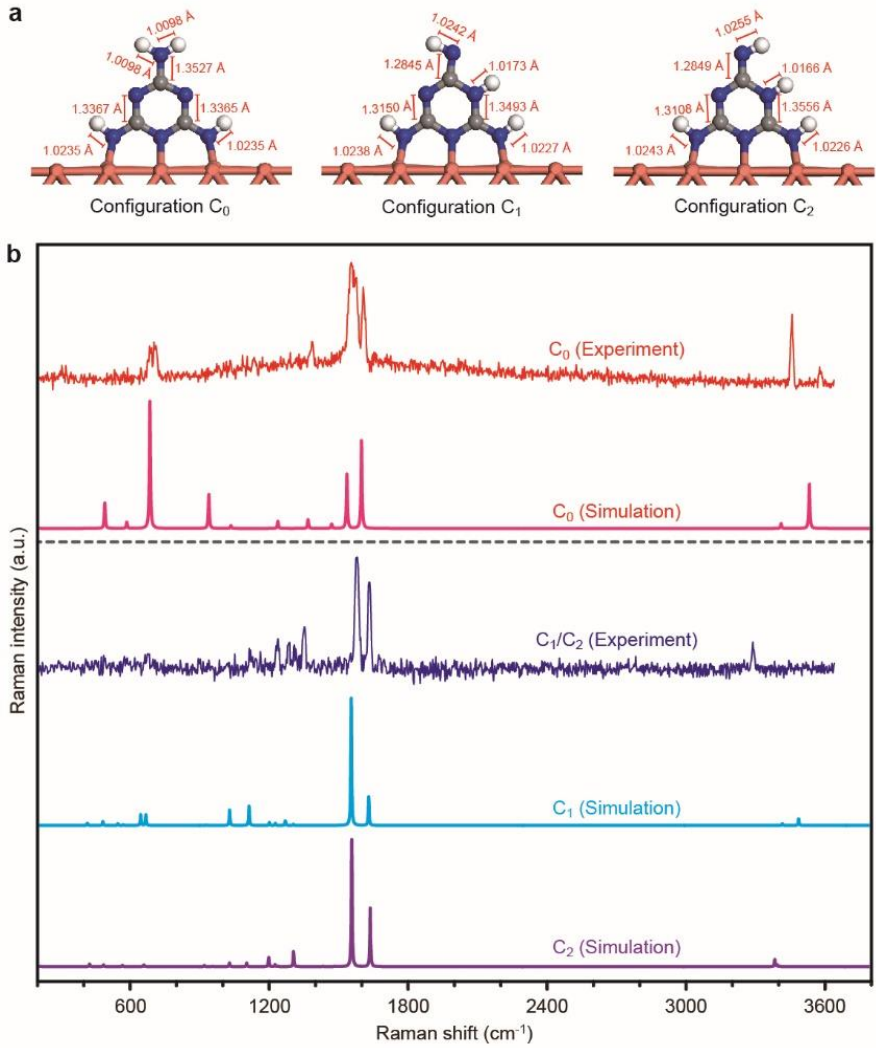

Fig. S5. Chemical structures and Raman spectra for configuration $\mathbf{C}_{0}, \mathbf{C}_{1}$ and $\mathbf{C}_{2}$. a, The optimized structures of adsorbed melamine molecule (configuration $\mathrm{C}_{0}$ ) and its tautomer structures 
(configurations $\mathrm{C}_{1}$ and $\mathrm{C}_{2}$ ). The typical bond lengths are labelled in each structure. $\mathbf{b}$, Comparison between the simulated Raman spectra and the experimental TERS spectra for configuration $\mathrm{C}_{0}, \mathrm{C}_{1}$ and $\mathrm{C}_{2}$, respectively.

For the two tautomerized configurations $\mathrm{C}_{1}$ and $\mathrm{C}_{2}$, because one of the top two hydrogen atoms in configuration $\mathrm{C}_{0}$ has transferred to the neighboring nitrogen atom, there are no symmetric and anti-symmetric vibrations of an $-\mathrm{NH}_{2}$ group anymore, consistent with the absence of Raman signals for such two vibrations and the emergence of only one Raman peak at $\sim 3297 \mathrm{~cm}^{-1}$ at one side of the cyanuric ring (position a in main-text Fig. 4e). Moreover, the appearance of a new Raman peak at $\sim 3308 \mathrm{~cm}^{-1}$ at the other side of the ring (position $a^{\prime}$ in main-text Fig. 4e) suggests the formation of a new $\mathrm{N}-\mathrm{H}$ bond in this side of cyanuric ring. The observed rapid oscillation between the two tautomers $\left(\mathrm{C}_{1}\right.$ and $\left.\mathrm{C}_{2}\right)$ is an indication of a weakened top $\mathrm{N}-\mathrm{H}$ bond, as evidenced by the red-shifting of its stretching vibrational frequency to $\sim 3297 \mathrm{~cm}^{-1}$ or $\sim 3290 \mathrm{~cm}^{-1}$ (in main-text Fig. 4e). Indeed, an elongation in the top $\mathrm{N}-\mathrm{H}$ bond length is observed for the optimized tautomer structures, about $0.014 \AA$ for configuration $\mathrm{C}_{1}$ and about $0.015 \AA$ for configuration $C_{2}$ (Fig. S5a). In this sense, although the tautomerized structure oscillates between two configurations, the high spectral sensitivity of TERS still enables us to specify each of them by positioning the tip at different sides, which in turn suggests that the top $\mathrm{N}-\mathrm{H}$ bond is in a flipping motion, rather than rotating. Similar bond-related analyses can be used for understanding the spectral evolution of other vibrational modes. As shown in main-text Fig. $4 \mathrm{~d}$, the $-\mathrm{NH}_{2}$ deforming mode at $1602 \mathrm{~cm}^{-1}$ for configuration $\mathrm{C}_{0}$ disappears in $\mathrm{C}_{1} / \mathrm{C}_{2}$ because there is no $-\mathrm{NH}_{2}$ group anymore due to the breaking of one $\mathrm{N}-\mathrm{H}$ bond at the top. The atop $\mathrm{C}-\mathrm{N}$ stretching mode at $1548 \mathrm{~cm}^{-1}$ in $\mathrm{C}_{0}$ is significantly blue-shifted to $1627 \mathrm{~cm}^{-1}$ due to the change of bonding characters from a single bond to a $\mathrm{C}=\mathrm{N}$ double bond (see Supplementary Movie 2 for vibrational animation), as evidenced by a greatly shortened bond length by $0.068 \AA$. By contrast, the $1570 \mathrm{~cm}^{-1}$ mode for $\mathrm{C}_{0}$ is only slightly blue-shifted to $1574 \mathrm{~cm}^{-1}$ because both of them are associated with the in-phase stretching vibrations of the $\mathrm{C}=\mathrm{N}$ bonds on the two sides of the cyanuric ring. The mode at 1380 $\mathrm{cm}^{-1}$ in $\mathrm{C}_{0}$ appears to be red-shifted to $1351 \mathrm{~cm}^{-1}$, which is related to out-of-phase stretching vibrations of the $\mathrm{C}=\mathrm{N}$ bonds on the two sides of the cyanuric ring. Notably, the emergence of new peaks at $1119,1235,1287$ and $1316 \mathrm{~cm}^{-1}$ is closely related to the breaking of old bonds and formation of new bonds. They are all associated with the in-plane rocking motions of both the newly formed $\mathrm{N}-\mathrm{H}$ bond on the ring side and the weakened $\mathrm{N}-\mathrm{H}$ bond at the top, although the relative amplitudes of atomic displacements are different. We would like to note that the surface selection rule is also valid for the $\mathrm{C}_{1} / \mathrm{C}_{2}$ species, as reflected by the two dominant peaks at 1574 $\mathrm{cm}^{-1}$ and $1627 \mathrm{~cm}^{-1}$, which are associated with the vibrations oriented along the tip axis and can be thus dramatically enhanced by the axial plasmonic field. (1) 

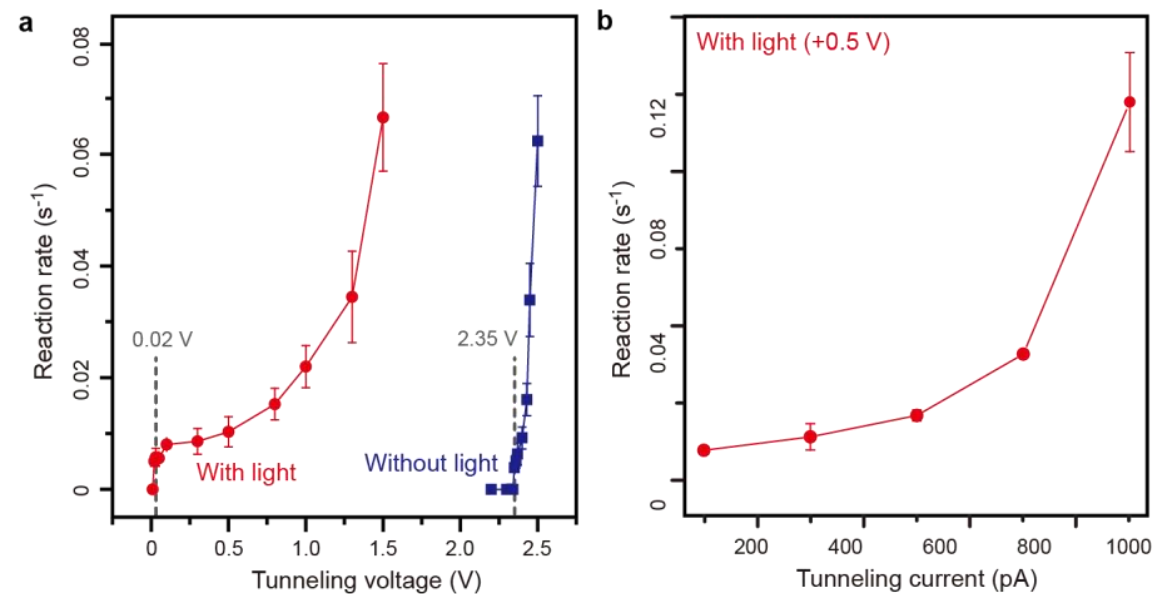

c

d
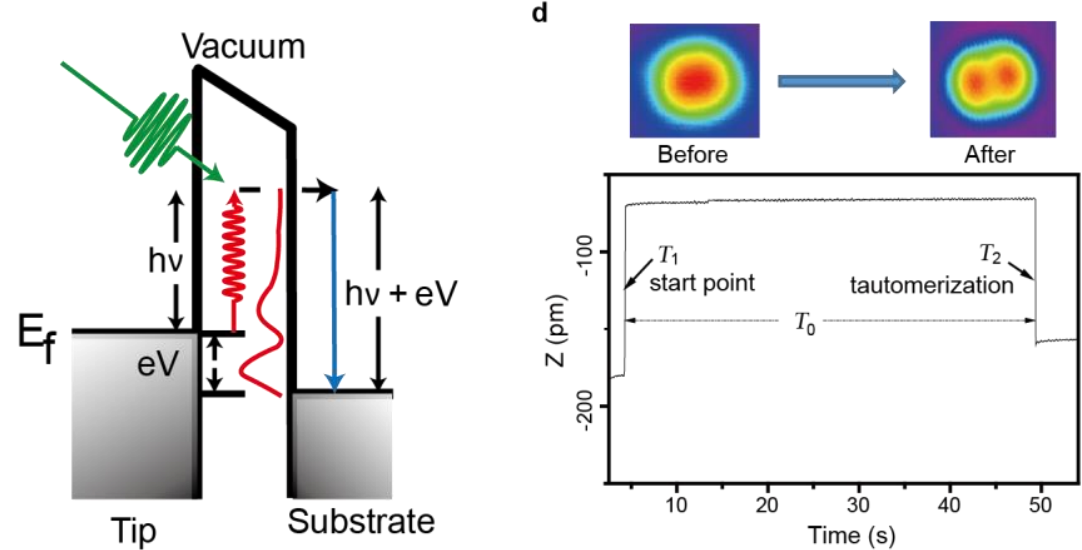

Fig. S6. Reaction rates for the hydrogen tautomerization from configuration $\mathbf{C}_{0}$ to $\mathbf{C}_{1} / \mathbf{C}_{2}$. $\mathbf{a}$, The reaction rate as a function of bias voltages with (red) or without (blue) $532 \mathrm{~nm}$ laser illumination. b, The reaction rate at different tunneling currents under a fixed bias voltage of $0.5 \mathrm{~V}$ with $532 \mathrm{~nm}$ laser illumination. c, Schematic diagram of possible hydrogen tautomerization mechanism. Such photoassisted tunneling mechanism is believed to be responsible for the hydrogen tautomerization under light illumination. ${ }^{11} \mathbf{d}$, The procedure of measuring reaction rates of tautomerization. We first placed the tip far away from a melamine- $\mathrm{C}_{0}$ molecule and changed the set point to the designated value. Then the tip was moved to the molecular center of melamine- $\mathrm{C}_{0}$, which we define as the time start point $T_{1}$. After that, we paid careful attention to the $Z$-history and watched until a sudden jump happened. This jump, defined as the time stop point $T_{2}$, indicates that the occurrence of tautomerization. Then we moved the tip away quickly to the $\mathrm{Cu}(100)$ surface nearby, changed the set point to the normal scanning condition (for instance, $-1 \mathrm{~V}, 2 \mathrm{pA}$ ) and scanned the same molecule again to confirm that the tautomerization indeed happened. The reciprocal of the time difference between $T_{1}$ and $T_{2}$ (namely $T_{0}$ ) is defined as the reaction rate of tautomerization under a certain set point. Every data point was measured at least three times and then statistically averaged.

Movie S1.

Animation for the representative vibrational modes of the chemisorbed melamine molecule (configuration $\mathrm{C}_{0}$ ) on $\mathrm{Cu}(100)$ that are assigned for the labeled peaks observed experimentally in main-text Fig. 1.

\section{Movie S2.}


353 Animation for the representative vibrational modes of two tautomers (configurations $\mathrm{C}_{1}$ and $\mathrm{C}_{2}$ ) 354 on $\mathrm{Cu}(100)$ that are assigned for the labeled peaks observed experimentally in main-text Fig. 4.

\section{$355 \quad$ References}

356 (1) Zhang, Y.; Yang, B.; Ghafoor, A.; Zhang, Y.; Zhang, Y.-F.; Wang, R.-P.; Yang, J.-L.; Luo, Y.; Dong, Z.-C.; Hou, J. G. Visually 357 Constructing the Chemical Structure of a Single Molecule by Scanning Raman Picoscopy. Natl. Sci. Rev. 2019, 6, 1169-1175.

358 (2) Kresse, G.; Furthmüller, J. Efficiency of Ab-Initio Total Energy Calculations for Metals and Semiconductors Using a 359 Plane-Wave Basis Set. Comput. Mater. Sci. 1996, 6, 15-50.

360 (3) Kresse, G.; Furthmüller, J. Efficient Iterative Schemes for Ab Initio Total-Energy Calculations Using a Plane-Wave Basis Set. $361 \quad$ Phys. Rev. B 1996, 54, 11169.

362 (4) Blöchl, P. E. Projector Augmented-Wave Method. Phys. Rev. B 1994, 50, 17953.

363 (5) Dion, M.; Rydberg, H.; Schröder, E.; Langreth, D. C.; Lundqvist, B. I. Van der Waals Density Functional for General 364 Geometries. Phys. Rev. Lett. 2004, 92, 246401.

365 (6) Klimeš, J.; Bowler, D. R.; Michaelides, A. Chemical Accuracy for the Van der Waals Density Functional. J. Phys. Condens. 366 Matter. 2009, 22, 022201.

367 (7) Hu, W.; Duan, S.; Zhang, G. P.; Ma, Y.; Tian, G. J.; Luo, Y. Quasi-Analytical Approach for Modeling of Surface-Enhanced 368 Raman Scattering. J. Phys. Chem. C 2015, 119, 28992-28998.

369 (8) Fonari, A. Stauffer, S. vasp_raman.py. 2013.

370 (9) Lee, J.; Crampton, K. T.; Tallarida, N.; Apkarian, V. A. Visualizing Vibrational Normal Modes of a Single Molecule with 371 Atomically Confined Light. Nature 2019, 568, 78-82.

372 (10) Socrates, G. Infrared and Raman Characteristic Group Frequencies: Tables and Charts. John Wiley \& Sons: 2004.

373 (11) Li, S.; Czap, G.; Wang, H.; Wang, L. K.; Chen, S. Y.; Yu, A.; Wu, R. Q.; Ho, W. Bond-Selected Photodissociation of Single 374 Molecules Adsorbed on Metal Surfaces. Phys. Rev. Lett. 2019, 122, 077401. 